\title{
Preclinical Studies of MSC-Derived Extracellular Vesicles to Treat or Prevent Graft Versus Host Disease: a Systematic Review of the Literature
}

\author{
Manika Gupta ${ }^{1,2} \cdot$ Alvin Tieu $^{2,3} \cdot$ Mitchell Slobodian $^{3} \cdot$ Risa Shorr ${ }^{4}$ - Dylan Burger ${ }^{2,5}$ - Manoj M. Lalu ${ }^{2,3}$. \\ David S. Allan ${ }^{1,2,3}$ (D)
}

Accepted: 12 October 2020 / Published online: 7 November 2020

(C) Springer Science+Business Media, LLC, part of Springer Nature 2020

\begin{abstract}
Introduction Treating and preventing graft-versus-host disease (GVHD) after allogeneic hematopoietic cell transplant (HCT) remains a significant challenge. The use of mesenchymal stromal cell-derived extracellular vesicles (MSC-EVs) appears promising and a systematic review of preclinical studies is needed to accelerate the design of translational studies.

Methods We identified 4 eligible studies from a systematic review performed on December 1, 2018. In brief, eligible studies included the treatment or prevention of GVHD in animal models and the use of MSC-EVs. Study design and outcome data were extracted and reporting was evaluated using the SYRCLE tool to identify potential bias.

Results Two studies assessed the efficacy of MSC-EVs in treatment of GVHD and 2 studies address prevention. Mice treated with MSC-EVs showed improved median survival, GVHD clinical scores and histology scores as compared to untreated mice with GVHD. Prophylactic treatment with MSC-EVs attenuated GVHD severity and improved median survival as compared to no treatment or saline.

Conclusion Our systematic review provides important insight regarding the potential of MSC-EVs to treat or prevent GVHD. Although few studies were identified, improved survival and attenuated histologic findings of GVHD were observed in mice after MSC-EV administration for the treatment and prevention of GVHD. Dosing of EVs and route of administration remain inconsistent, however, and scalability of EV isolation for clinical studies remains a challenge. Standardized outcome reporting is needed to pool results for metanalysis.
\end{abstract}

Keywords Graft versus host disease $\cdot$ Extracellular vesicles $\cdot$ Preclinical $\cdot$ Systematic review $\cdot$ Mesenchymal stromal cells

\section{Introduction}

Special Issue on Exosomes and Microvesicles: from Stem Cell Biology to Translation in Human Diseases

Guest Editor: Giovanni Camussi

David S. Allan

daallan@toh.ca

1 Department of Medicine (Blood and Marrow Transplantation), The Ottawa Hospital, 501 Smyth Rd, Box 704, Ottawa, ON K1H 8L6, Canada

2 Faculty of Medicine, University of Ottawa, Ottawa, ON, Canada

3 Clinical Epidemiology, Ottawa Hospital Research Institute, Ottawa, ON, Canada

4 Library Services, The Ottawa Hospital, Ottawa, ON, Canada

5 Chronic Disease Programs, Ottawa Hospital Research Institute, Ottawa, ON, Canada
Graft-versus-host disease (GVHD) is a common and serious complication of allogeneic hematopoietic cell transplant (HCT). GVHD involves immune cells from the donor recognizing the host and initiating an immune reaction that causes disease in transplant recipients. The overall cumulative incidence of GVHD is estimated to be $40 \%$ in patients receiving sibling donor transplants and up to $60 \%$ in patients receiving unrelated donor transplants $[1,2]$. While improved methods of preventing GVHD are under study, risk factors for GVHD include degree of HLA disparity, intensity of the transplant conditioning regimen, GVHD prophylactic regimen, source of graft cells, donor and recipient gender disparity, and the hematopoietic cell transplantation-specific comorbidity index [3-5]. Patients with either acute or chronic GVHD that does not respond to corticosteroids have high levels of morbidity 
and mortality related to intense immune suppression and/or end-organ damage from progression of GVHD. Despite many clinical studies evaluating treatment interventions, there are very few approved therapies for steroid-resistant GVHD [6].

Donor T lymphocytes that recognize recipient major and/or minor histocompatability antigens can be activated by recipient antigen-presenting cells following injury to host tissues caused by the pre-transplant chemo-radiotherapy conditioning, leading to cell-mediated and inflammatory cytokinemediated tissue damage. Both cytotoxic $\mathrm{T}$ cells and natural killer cells can be activated and mediate target cell lysis via the Fas/FasL pathway and by inflammatory cytokines produced by $\mathrm{T}$ cells that exacerbate tissue injury in skin, liver and gut epithelia [7]. FoxP3-expressing regulatory $\mathrm{T}$ cells and other immune regulatory cells such as natural killer cells and mesenchymal stromal cells (MSCs), can reduce inflammation and are associated with tolerance induction and reducing the severity of GVHD [8]. MSCs can attenuate immune and inflammatory responses in many animal models, including GVHD. MSCs can influence both innate and adaptive immune responses by releasing factors such as IL-6 to inhibit dendritic cell maturation, and by transforming growth factor beta, indoleamine 2,3-dioxygenase (IDO), nitric oxide, and prostaglandin 2 to reduce $\mathrm{T}$ cell effector functions [9]. Moreover, IDO can induce apopotosis of $\mathrm{T}$ effector cells and promote differentiation of regulatory $\mathrm{T}$ cells [10]. MSCs can also down-regulate factors expressed by $\mathrm{T}$ cells to inhibit their migration and they express checkpoint inhibitors which further enhances their immune suppressive functions [11]. Moreover, MSCs have been evaluated in several clinical studies for the treatment or prevention of GVHD [12-14]. Although MSCs have been approved for the treatment of steroid-resistant acute GVHD in a limited number of jurisdictions, their efficacy remains highly variable and is hampered by marked heterogeneity between studies [14]. The conditioned media of MSC cultures has been demonstrated to account for a significant portion of therapeutic responses in animal studies, likely owing to extracellular vesicles (EVs) that are present in the media [15].

EVs include exosomes and microvesicles that are released from cells and can be small or large in size, in accordance with recent nomenclature adopted by the International Society for Extracellular Vesicles (ISEV) [16]. Exosomes are approximately $50-150 \mathrm{~nm}$ in size and originate from endosomes while microvesicles are approximately $150-1000 \mathrm{~nm}$ in size and originate from plasma membrane [17]. EVs contain bioactive signaling molecules such as microRNA, mRNA and chemokines from within MSCs which can alter gene expression of target cells, whether pathologic or normal cells, to modulate immune and inflammatory responses. One case report describes a patient treated with the exosome-enriched fraction from MSC supernatants who had significant improvement in inflammatory markers and clinical improvement in
GVHD of the gut, mucosa and skin [18]. Other clinical reports for GVHD, however, are lacking. Various pre-clinical studies have explored the therapeutic potential of MSC-EVs in the treatment of GVHD in the context of allogeneic HCT. These studies have used different methods to culture MSCs, isolate EVs, and have tested these cell-based therapies in different animal models using different endpoint measurements. There is a need to perform a systematic review to identify the extent to which MSC-EVs are effective in treating or preventing GVHD and to inform potential future preclinical and clinical studies regarding the optimal method of isolating and administering EVs, including aspects such as dosing and route of administration and schedules of EV treatment.

Our recent systematic review of all preclinical studies of MSC-EVs in animal models of various types of organ dysfunction and injury identified more than 200 studies. A subset of these studies addressed the role of MSC-EVs to treat or prevent GVHD [19]. A more complete analysis of data from these studies is needed to gain insight regarding the animal models used to study GVHD, methods of isolating EVs and their robust characterization that will be critical for regulatory approval and dosing regimens. The insight gained from a systematic analysis of these preclinical studies will inform future studies aimed at translating this promising treatment modality into the clinical realm.

\section{Methods}

We identified eligible studies from a comprehensive systematic scoping review performed in May 2018 and updated in December 2018 [20] (registered April 12, 2019 on PROSPERO at www.crd.york.ac.uk/prospero, protocol: CRD42019123918). In brief, this larger scoping review included all studies using MSC-EVs in preclinical animal models of disease, organ injury, tumor growth or altered immune response. Studies without a comparator arm (such as vehicle control, placebo, MSCs, or fibroblast EVs) were excluded. Relevant studies associated with GVHD were identified for further data extraction and analysis in this report (PROSPERO protocol CRD42020146580; registered March 7, 2020).

A data extraction tool was developed to capture relevant data including mode of induction of GVHD in mouse models, method of isolation of MSC-EVs and treatment related outcome data. The outcomes measured included median survival (days), GVHD clinical scores, skin scores, qualitative and quantitative histology assessment, fibrosis percentage and animal weight. Data extraction was completed using the information software, DistillerSR (Evidence Partners, Ottawa Canada) on all included studies. Preclinical study design and reporting was evaluated using the Systematic Review Centre 
for Laboratory Animal Experimentation (SYRCLE) tool to identify and report on potential bias in preclinical studies [21].

MSC characterization was assessed according to the minimal criteria established by the International Society for Cellular Therapy [22]. Specifically, we assessed whether studies reported if MSCs were (1) plastic adherent in standard culture conditions; (2) expressed the cell surface markers CD105, CD73, CD90 and lacked expression of CD45, CD34, CD14 or CD11b, CD79a, and HLA-DR surface molecules; and (3) differentiated to osteoblasts, adipocytes and chondroblasts in vitro.

\section{Results}

In our recent comprehensive systematic scoping review of methods and outcome reporting in all preclinical studies of MSC-derived EVs, a total of 208 studies were identified [19, 20] and an updated search conducted December 2018 identified 284 additional records that underwent screening for potential relevance and eligibility, identifying 113 additional studies. Amongst these 321 studies, four addressed GVHD treatment and/or prevention and were included in the quantitative and indepth analysis described in this report (see Fig. 1). Two studies assessed the efficacy of MSC-derived EVs as treatment of GVHD [23, 24] while 2 studies assessed the efficacy of MSC- derived EVs to prevent GVHD $[25,26]$. The main characteristics of these studies are summarized in Table 1.

\section{Risk of Bias}

We utilized the SYRCLE tool [21] to assess risk of bias in selected pre-clinical animal intervention studies. Random allocation of animals to experimental and control groups was described for only one of the four studies. None of the studies reported blinding during randomization or outcome assessments. Baseline characteristics of mice were reported for three of the four studies. None of the studies reported initial number of mice who underwent transplant and none of the studies reported sample size calculations. It was not clear in any of the studies whether there was an attrition bias and whether all treated animals were included in the final analysis. Table 2 summarizes the assigned risk of bias in each of the domains for studies included in our analysis.

\section{Isolation and Characterization of MSC- EVs}

The source of cells used to manufacture MSCs was human bone marrow ( 2 studies), human embryonic stem cells (1 study) and human umbilical cord blood (1 study). All 4 studies utilized the Dominici criteria [22] to define MSCs (see Table 1 ). The use of early passage cells (passage 3 ) was
Fig. 1 PRISMA diagram outlining the identification of articles included in the analysis
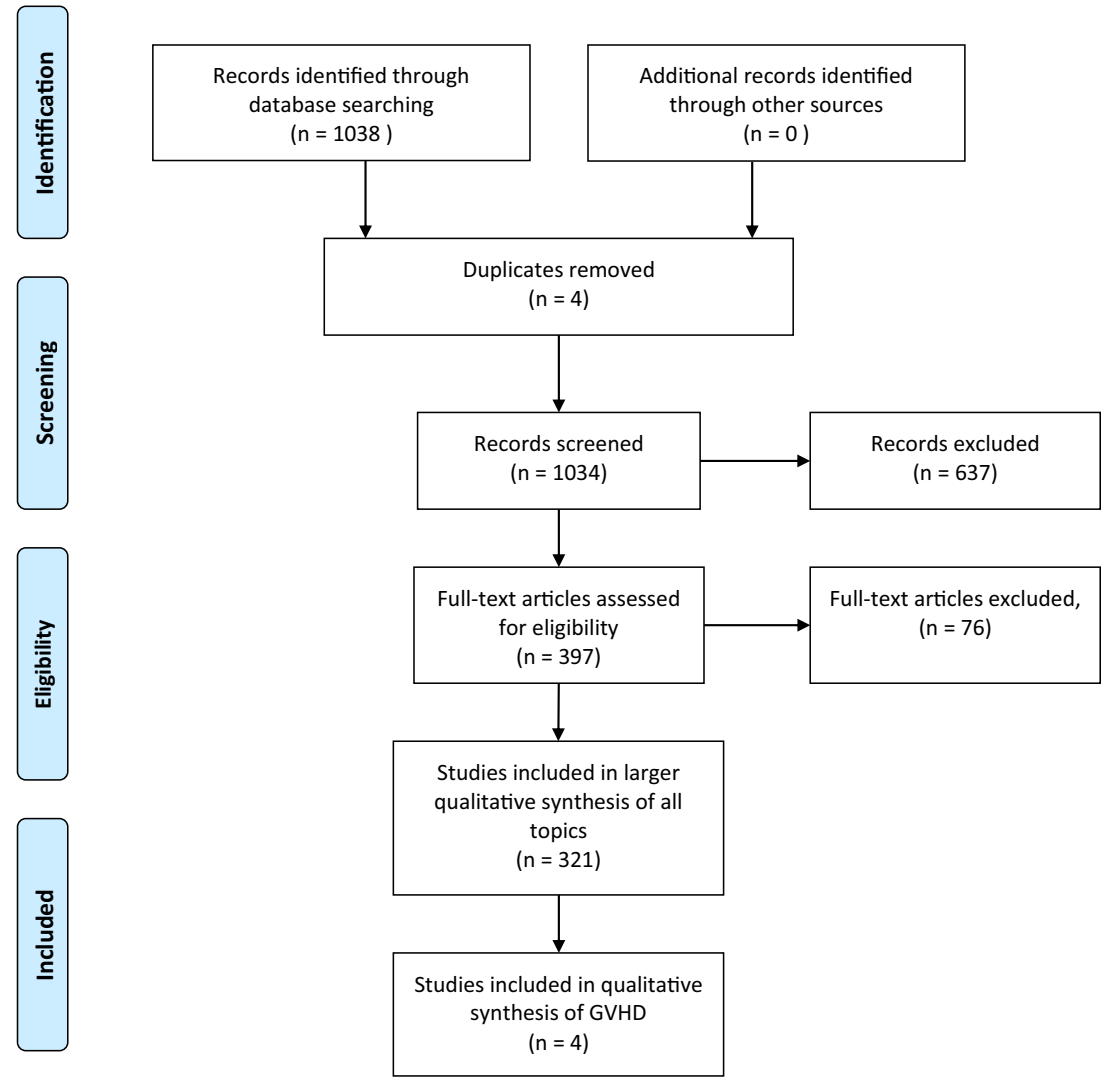


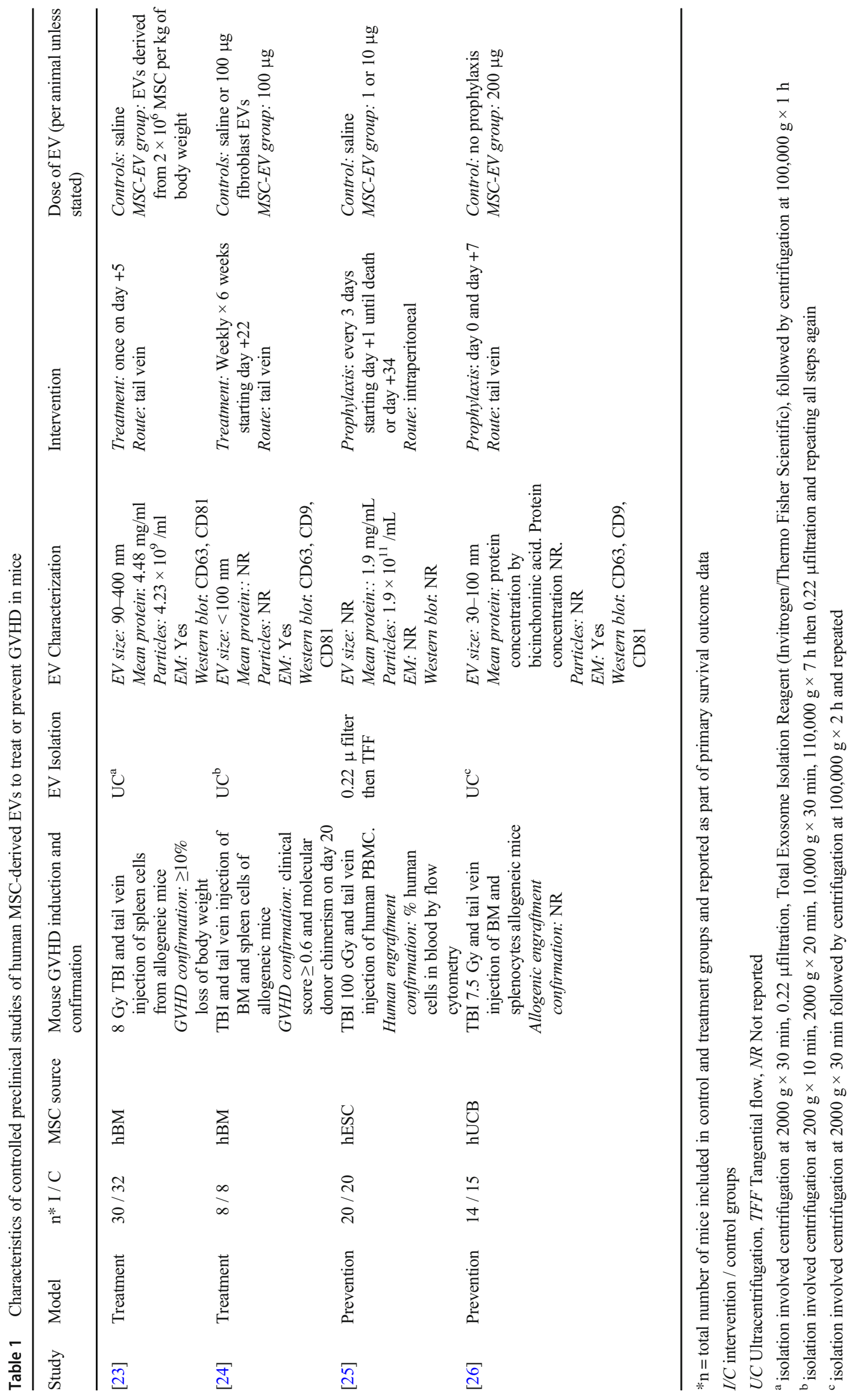


Table 2 Risk of bias using SYRCLE tool for preclinical studies. Low risk (open circles), high risk (black) and unclear risk of bias (grey) are indicated for each study

\begin{tabular}{|c|c|c|c|c|c|c|c|c|c|c|}
\hline & \multicolumn{3}{|c|}{ Selection bias } & \multicolumn{2}{|c|}{ Performance bias } & \multicolumn{2}{|c|}{ Detection bias } & $\begin{array}{c}\text { Attrition } \\
\text { bias }\end{array}$ & \multicolumn{2}{|l|}{$\begin{array}{c}\text { Reporting } \\
\text { bias }\end{array}$} \\
\hline Study & 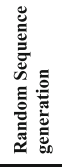 & 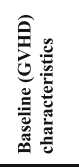 & 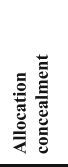 & 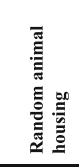 & 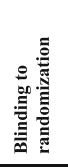 & 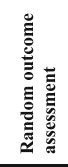 & 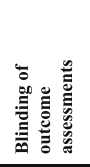 & 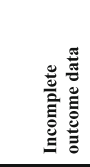 & 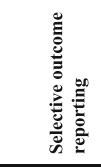 & 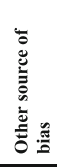 \\
\hline$[23]$ & $\mathrm{O}$ & $\bullet$ & $\bullet$ & $\bullet$ & $\bullet$ & $\bullet$ & $\bullet$ & - & $\mathrm{O}$ & $\mathrm{O}$ \\
\hline [24] & $\bullet$ & O & $\bullet$ & $\bullet$ & - & - & • & - & $\mathrm{O}$ & $\mathrm{O}$ \\
\hline [25] & $\bullet$ & $\mathrm{O}$ & $\bullet$ & $\bullet$ & $\bullet$ & $\bullet$ & $\bullet$ & - & $\mathrm{O}$ & $\mathrm{O}$ \\
\hline [26] & $\bullet$ & $\mathrm{O}$ & - & $\bullet$ & $\bullet$ & $\bullet$ & - & - & $\mathrm{O}$ & $\mathrm{O}$ \\
\hline
\end{tabular}

explicitly reported in one study (24). None of the studies reported MSC viability at the time of EV isolation. Three studies $(23,25,26)$ cultured MSCs in serum-free media for $24-72 \mathrm{~h}$ and one study (24) used "exosome-free" fetal bovine serum for $48 \mathrm{~h}$ prior to $\mathrm{EV}$ isolation. Three of the four studies isolated EVs using ultracentrifugation, characterized EVs using immunoblotting for anti-CD63, CD81 and/or CD9 antibodies (no studies included non-EV associated antigens as negative controls) and examined EV shape and size using electron microscopy [23, 24, 26]. One study utilized tangential flow filtration for isolation of EVs [25]. More details of the isolation methods, including additional centrifugation steps are included in Table 1. There was heterogeneity in the size of EVs amongst the studies ranging from 30 to $400 \mathrm{~nm}$. (Table 1).

\section{Mouse GVHD Model Characteristics}

Although the mechanism of GVHD induction varied, in general the studies involved total body irradiation (TBI) followed by tail vein injection of allogenic bone marrow or splenocytes to induce GVHD in mice. One of the four studies utilized a xenograft model which involved injection of human BM to induce GVHD. For treatment studies, GVHD was confirmed based on clinical score or weight loss prior to therapy. For the prevention studies, confirmation of engraftment of the transplanted cells was confirmed and reported in one of two studies.

The baseline characteristics of mice with GVHD were examined in each of the studies. Specifically, age (age 7 to 12 weeks old, reported in 3 studies), gender (all female in 1 study; all male in 1 study; not reported in 2 studies), weight (reported in 1 study), GVHD score (reported in 3 studies) and skin score (reported in 1 study) were provided as baseline characteristics. The baseline GVHD score incorporates characteristics such as skin integrity, body weight, hunch and fur texture and was not statistically different between the intervention groups in the 3 studies using this score [24-26].

\section{Relative Heterogeneity in Outcome Reporting}

All of the studies reported median survival and GVHD clinical scores following treatment (Table 3). There was heterogeneity, however, in the reporting of skin scores (2/4), histology scores (1/4), fibrosis percentage (1/4), qualitative histology assessment (3/4) and weight (1/4). Furthermore, criteria for GVHD scoring, histology scores and skin scores varied between studies (Table 3).

\section{Mice with GVHD Treated with MSC-EVs}

The pre-clinical studies by Lai et al. [24] and Fujii et al. [23] utilized human BM derived MSC-EVs for treatment of GVHD. Differences in the mean size and/or size distribution of EVs used in these studies are provided in Table 1. Improvement in primary outcomes including median survival and GVHD clinical scores were noted in both studies (Table 4). MSC-EV treatment was associated with apparent benefit in median survival in both studies compared to control mice with GVHD (48 days vs 34 days, $p<0.05$ [24]; 16 days vs 10 days, $p<0.01$ [23]) (Table 4). In addition, GVHD scores were improved ( 3 vs 6 points of maximum score of 7.9, $p<0.05)$ measured at 50 days post-transplant in the Lai et. al study [24] and were improved in the Fujii et. al study [23] at 10 days after transplant (2.8 vs 3.5, maximum score of $10 ; p$ value $<0.01$ ) for $E V$ treated mice compared with controls. See Table 4 for a summary of reported outcomes.

In addition, some secondary outcomes including histology parameters and skin scores were improved with treatment in both studies. Qualitative histology parameters as well as percentage of fibrosis for liver, lung and skin were improved in mice treated with EVs as compared to control in the Lai et. al study [24]. Fujii et al. [23] showed improved histology scores 
Table 3 Outcomes reported in preclinical studies of treatment (light grey) and prevention (dark grey) of GVHD

\begin{tabular}{|c|c|c|c|c|c|c|c|}
\hline Study & 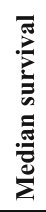 & 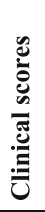 & 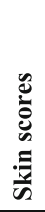 & 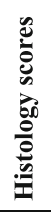 & 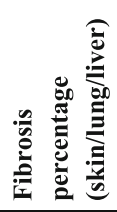 & 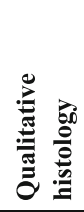 & $\begin{array}{l}\vec{z} \\
\overrightarrow{0} \\
\overrightarrow{0}\end{array}$ \\
\hline [23] & $\sqrt{ }$ & $\sqrt{ }$ & $\sqrt{ }$ & $\sqrt{ }$ & & $\sqrt{ }$ & \\
\hline [24] & $\sqrt{ }$ & $\sqrt{ }$ & $\sqrt{ }$ & & $\sqrt{ }$ & $\sqrt{ }$ & \\
\hline [25] & $\sqrt{ }$ & $\sqrt{ }$ & & & & & \\
\hline [26] & $\sqrt{ }$ & $\sqrt{ }$ & & & & $\sqrt{ }$ & $\sqrt{ }$ \\
\hline
\end{tabular}

in large bowel, however, no difference in histology scores were observed in liver and small bowel samples with MSCEV treatment compared to controls (see Table 4).

\section{MSC-EVs as Prevention for GVHD}

The pre-clinical studies by Zhang et al. [25] and Wang et al. [26] studied the effect of MSC-derived EVs on prevention of GVHD. Both studies demonstrated that prophylactic treatment with MSC-EVs attenuated GVHD severity and improved median survival as compared to controls ( 60 days vs 36 days ( $p<0.01$ [26]); 25 days vs 22 days $(p<0.05)$ [25]) (Table 4). In the study by Wang et. al, [26] prophylaxis with MSC-EVs reduced the severity of GVHD manifestations at 28 days following transplantation, and was associated with less weight loss $(p<0.001)$, improved
GVHD score $(p<0.001)$ and prolonged median survival $(p<0.01)$ compared to controls. In addition, histology scores for GVHD-associated changes were reduced in the group treated with MSC-EVs.

\section{Discussion}

Our systematic review of preclinical studies on the use of MSC-EVs to treat or prevent GVHD highlights the potential of this novel cell-free therapy. Although only a small number of studies were identified, the studies consistently show improved overall survival and GVHD scores with the use of MSC-EVs for both treatment and prevention of GVHD. All studies utilized an established GVHD mouse model and EVs

Table 4 Outcomes for preclinical models of treatment or prevention of GVHD

\begin{tabular}{|c|c|c|c|c|c|}
\hline Study & Model & Survival (I vs C) & GVHD Clinical score (I vs C) & Skin score (I vs C) & Histology (I vs C) \\
\hline [23] & Treatment & 16 vs $10 \mathrm{~d}(p<0.01)$ & 2.8 vs 3.5 at $10 \mathrm{~d}(p<0.01)^{\mathrm{a}}$ & Severe, $60 \%$ vs $89 \%$ & $\begin{array}{l}\text { Large bowel }{ }^{\mathrm{d}}: 7 \text { vs } 12(p<0.01) \text { at } 6 \\
\text { Liver/small bowel: no difference at } 6\end{array}$ \\
\hline [24] & Treatment & 48 vs 34 d (p NR) & 3 vs 6 at $50 \mathrm{~d}(\mathrm{p} \mathrm{NR})^{\mathrm{b}}$ & 2.5 vs 3.5 at $50 \mathrm{~d}(\mathrm{p} \mathrm{NR})^{\mathrm{c}}$ & $\begin{array}{l}\text { Liver, lung, skin: Improved } \\
\text { Fibrosis: Liver: } 5 \% \text { vs } 20 \%(p<0.05) \\
\text { Lung: } 6 \% \text { vs } 15 \%(p<0.05) \\
\text { Skin: } 30 \% \text { vs } 75 \%(p<0.01)\end{array}$ \\
\hline [25] & Prevention & 25 vs $22 \mathrm{~d}(p<0.05)$ & 4 vs 8 at $24 \mathrm{~d}(p<0.05)^{\mathrm{e}}$ & NR & NR \\
\hline [26] & Prevention & 60 vs $36 \mathrm{~d}(p<0.01)$ & 2 vs 10 at 56 days $(p<0.001)^{\mathrm{f}}$ & NR & Liver, large intestine, skin: Improved \\
\hline
\end{tabular}

${ }^{\text {a }}$ Clinical score at 10 days: 0 (good) to 2 (poor) for weight loss, posture, activity, fur texture and skin integrity and summed for a total score of $0-10$

${ }^{\mathrm{b}}$ Clinical score: Skin, body weight and hunch. Maximum total score $=7.9$

${ }^{\mathrm{c}}$ Skin score: Minimum score 0; maximum score 3.9

${ }^{\mathrm{d}}$ Histology score: Maximum total score of 28 for the large bowel and small bowel, and 40 for the liver

${ }^{\mathrm{e}}$ Mean combined disease activity index: Grade 0/1/2 assigned to weight loss, posture, activity, fur texture, skin integrity and paleness. Maximum total score $=1$

${ }^{\mathrm{f}}$ Clinical score: Grade 0/1/2 assigned to weight loss, posture, activity, fur texture and skin integrity. Maximum total score $=10$ 
were isolated from well characterized MSCs that met internationally recognized standards [22]. All of the studies characterized EVs using a combination of size, protein content, electron microscopy and/or surface marker expression. Heterogeneity, however, was observed amongst the studies with regard to EV size, dosing and treatment schedules, route of administration and scoring of GVHD. Larger, more definitive preclinical studies using standard characterization approaches and aligned outcome measures should help define the potential efficacy of MSC-EVs in the prevention or treatment of GVHD. Translation to clinical studies should be accelerated given the potential promise and benefits of MSCEVs in this setting.

While most studies in our review used ultracentrifugation to isolate EVs, there may be some negative effects of using this approach. Ultracentrifugation uses very high forces in excess of $100,000 \times \mathrm{g}$ for prolonged periods which could damage EVs and this method has been associated with contamination by non-EV cellular material and reduced concentration of packaged RNA compared with other isolation methods which could impact therapeutic efficacy [27]. Although ultracentrifugation remains the most common approach to EV isolation, it may not be ideally suited to largescale production. Interest in other methods such as tangential flow filtration, used by one of the studies included in our review, represents a relatively easy method of isolating small EVs that are not complexed with other molecules and represents a procedure that may be less likely to physically alter EVs [28].

Regardless of isolation methods used to generate the cellderived product used in the studies, the origin of EVs within MSCs can also impact their contents and therapeutic effects on target cells. Small EVs (<150 nm [17]), or exosomes, are formed by late endosomes and a process of inward budding of multivesicular bodies and have surface markers which correspond to markers of the endosomal pathway (e.g. CD63, CD81, TSG101 and Alix). Large EVs $(150-1000 \mathrm{~nm}$ [17]), or microvesicles, are formed by outward budding of the plasma membrane and contain contents of the cytoplasm that are distinct from small EVs and may serve as biomarkers of cellular stress rather than effectors of immune modulation. Indeed, MSC-derived microvesicles have demonstrated promise as biomarkers of GVHD [29, 30]. Isolation methods such as centrifugation may yield MSC-derived products with a mixture of small and large EVs. The information provided in the reports rely heavily on size and cell surface marker expression to characterize EVs but more insight on the origin and profile of contents would add additional insight on their immune modulatory potential. As new studies emerge, greater adherence to the nomenclature and characterization recommended by the ISEV [16] will reduce heterogeneity between studies.

The studies identified in our review all used human-derived MSCs although the various tissue sources included bone marrow, umbilical cord blood and embryonic stem cells. The tissue origin of MSCs may impact the cargo within EVs and could account for different therapeutic effects. MSCs derived from bone marrow as compared with cord blood, for example, exert a greater influence on bone growth and differentiation, as recently summarized by multiple groups $[31,32]$. These groups describe secretomes that are distinct to the tissue of origin of MSCs which could also extend to the content of EVs derived from these cells. Adipose tissue-derived MSCs, however, appear to maintain similar immune modulatory function compared to bone marrow-derived MSCs [33]. In addition to tissue source, specific culture conditions can also impact EV content and their effects on target cells. Hypoxic culture conditions, for instance, can activate signaling pathways in MSCs to enrich EV content of specific molecules that augment their capacity to promote angiogenesis and facilitate tissue repair in animal models [34, 35]. Hypoxia can also enhance immune modulation by MSCs through increased secretion of indoleamine-pyrrole 2,3-dioxygenase and increased regulatory $\mathrm{T}$ cell induction [36]. We are not aware of studies that have compared human to mouse MSC-EVs, however, it is likely that the species of origin could also affect the contents of EVs and their therapeutic properties. The use of human tissues retains clinical relevance, however, which addresses a potential barrier for translation to human trials.

We are aware of only a single case report of a patient with steroid-refractory acute GVHD following allogeneic hematopoietic cell transplantation who received MSC-derived "exosomes" [18]. While a positive response was reported with a reduction in concomitant corticosteroid dosage, the patient died of pneumonia several months after receiving treatment. We are unaware of other clinical reports describing patients who received MSC-derived EVs for the treatment or prevention of GVHD. In a recent search of registered clinical trials (clinicaltrials.gov, search March 31,2020), we identified one study addressing the role of MSC-EVs to treat dry eye in patients with ocular GVHD (NCT04213248). Other registered clinical trials of MSC-EV include studies addressing the tolerance of inhalation in healthy volunteers (NCT04313647), treatment in patients with severe infection with SARS-cov-2 infection (NCT04276987), treatment of macular holes (NCT03437759), patients with acute ischemic stroke (NCT03384433), pre-term neonates with bronchopulmonary dysplasia (NCT03857841), type 1 diabetes mellitus (NCT02138331), epidermolysis bullosa (NCT04173650), and patients with pancreatic cancer harboring KRAS mutations and MSC-EVs that express short inhibitory RNA against the same KRAS mutation (NCT03608631).

The risk of bias assessment revealed multiple sources of potential bias in each of the studies. In particular, the lack of blinding was observed in all studies and allocation concealment was not reported in any study. Increasingly, it is recognized that reducing potential bias in preclinical studies will accelerate meaningful translation of effective therapies to the clinical 
domain while identifying interventions with limited efficacy and/or toxicity that should not be pursued further [37]. While heterogeneity in studies, small number of studies and overall modest number of animals limited our ability to pool data for a metanalysis, we were able to identify aspects of preclinical study design that could be addressed to reduce potential bias and allow for pooled estimates of efficacy in the future. Specifically, we recommend that future preclinical studies include randomization, blinding and allocation concealment in their study design, continue to use well characterized MSCs and EVs in accordance with international consensus guidelines from the ISCT and ISEV [16, 22], respectively, and use dosing and administration schedules that are consistent and applicable in a clinical setting. Recording standardized outcome measure of GVHD will allow pooling of data between studies.

Our study's limitations include the possibility of omitting published reports. Conference abstracts were not included as methodological details are often lacking. Given the overall small number of studies and modest number of animals contributing to results in the studies included in our analysis, larger more definitive studies are still needed. Reported outcomes were inconsistent and recorded at variable time points after the intervention. While heterogeneity in studies limited our ability to pool data for a metanalysis, we were able to identify aspects of preclinical study design that could be addressed to reduce potential bias and to allow for pooled estimates of efficacy in the future.

In conclusion, our systematic review provides a novel perspective regarding preclinical evidence supporting the use of MSC-EVs to treat and prevent GVHD. The studies show improved overall survival and GVHD clinical scores with the use of MSC-EVs in both treatment and prevention settings and improvements in histology of affected organs support the role of MSC-EVs in addressing the inflammatory damage caused by GVHD. Our work should facilitate definitive preclinical studies that can further define the benefits of MSCEVs in GVHD while accelerating the design of translational studies in patients. Standardized approaches to MSC and EV characterization are largely addressed in preclinical studies to date, and aspects of study design such as dosing, route and schedule of administration can be aligned along with standard approaches to outcome reporting. MSC-EVs appear promising for the treatment and prevention of GVHD and efforts to accelerate further research are encouraged.

Acknowledgements We acknowledge funding support from Canadian Institutes of Health Research (Vanier Graduate Scholarship) for AT and from The Ottawa Hospital Foundation for MS.

\section{Compliance with Ethical Standards}

The authors have no potential conflicts of interest to disclose. All results and data used in our analysis were obtained from published articles, therefore, approval from our institutional research ethics board regarding research involving human participants and/or animals and informed consent is not applicable.

Conflict of Interest None of the authors have conflicts of interest to declare.

\section{References}

1. Jagasia, M., Arora, M., Flowers, M. E. D., Chao, N. J., McCarthy, P. L., Cutler, C. S., Urbano-Ispizua, A., Pavletic, S. Z., Haagenson, M. D., Zhang, M. J., Antin, J. H., Bolwell, B. J., Bredeson, C., Cahn, J. Y., Cairo, M., Gale, R. P., Gupta, V., Lee, S. J., Litzow, M., Weisdorf, D. J., Horowitz, M. M., \& Hahn, T. (2012). Risk factors for acute GVHD and survival after hematopoietic cell transplantation. Blood., 119, 296-307.

2. Arai, S., Arora, M., Wang, T., Spellman, S. R., He, W., Couriel, D. R., Urbano-Ispizua, A., Cutler, C. S., Bacigalupo, A. A., Battiwalla, M., Flowers, M. E., Juckett, M. B., Lee, S. J., Loren, A. W., Klumpp, T. R., Prockup, S. E., Ringdén, O. T., Savani, B. N., Socié, G., Schultz, K. R., Spitzer, T., Teshima, T., Bredeson, C. N., Jacobsohn, D. A., Hayashi, R. J., Drobyski, W. R., Frangoul, H. A., Akpek, G., Ho, V. T., Lewis, V. A., Gale, R. P., Koreth, J., Chao, N. J., Aljurf, M. D., Cooper, B. W., Laughlin, M. J., Hsu, J. W., Hematti, P., Verdonck, L. F., Solh, M. M., Norkin, M., Reddy, V., Martino, R., Gadalla, S., Goldberg, J. D., McCarthy, P., PérezSimón, J. A., Khera, N., Lewis, I. D., Atsuta, Y., Olsson, R. F., Saber, W., Waller, E. K., Blaise, D., Pidala, J. A., Martin, P. J., Satwani, P., Bornhäuser, M., Inamoto, Y., Weisdorf, D. J., Horowitz, M. M., Pavletic, S. Z., \& Graft-vs-Host Disease Working Committee of the CIBMTR. (2015). Increasing incidence of chronic graft-versus-host disease in allogeneic transplantation: a report from the Center for International Blood and Marrow Transplant Reseach. Biology of Blood and Marrow Transplantation, 21, 266-274.

3. Arora, M., Hemmer, M. T., Ahn, K. W., Klein, J. P., Cutler, C. S., Urbano-Ispizua, A., Couriel, D. R., Alousi, A. M., Gale, R. P., Inamoto, Y., Weisdorf, D. J., Li, P., Antin, J. H., Bolwell, B. J., Boyiadzis, M., Cahn, J. Y., Cairo, M. S., Isola, L. M., Jacobsohn, D. A., Jagasia, M., Klumpp, T. R., Petersdorf, E. W., Santarone, S., Schouten, H. C., Wingard, J. R., Spellman, S. R., Pavletic, S. Z., Lee, S. J., Horowitz, M. M., \& Flowers, M. E. D. (2015). Center for International Blood and Marrow Transplant Research graft-versushost disease risk score predicts mortality in an independent validation cohort. Biology of Blood and Marrow Transplantation, 21, 640-645.

4. Hahn, T., McCarthy, P. L., Zhang, M. J., et al. (2008). Risk factors for acute graft-versus-host disease after human leukocyte antigenidentical sibling transplants for adults with leukemia. Journal of Clinical Oncology, 26, 5728-5734.

5. Flowers, M. E., Inamoto, Y., Carpenter, P. A., Lee, S. J., \& Kiem, H. P. (2011). Comparative analysis of risk factors for acute graftversus-host disease according to National Institutes of Health consensus criteria. Blood., 117, 3214-3219.

6. Shapiro, R.M., \& Antin, J.H. (2020). Therapeutic options for steroid-refractory acute and chronic GVHD: an evolving landscape. Expert Review of Hematology. epub ahead of print April 5, 2020.

7. Ghimire, S., Weber, D., Mavin, E., Wang, X. N., Dickinson, A. M., \& Holler, E. (2017). Pathophysiology of GvHD and other HSCTrelated major complications. Frontiers in Immunology, 8, 79.

8. Blazar, B. R., MacDonald, K. P. A., \& Hill, G. R. (2018). Immune regulatory cell infusion for graft-versus-host disease prevention and therapy. Blood., 131, 2651-2660. 
9. Ren, G., Zhang, L., Zhao, X., Xu, G., Zhang, Y., Roberts, A. I., Zhao, R. C., \& Shi, Y. (2008). Mesenchymal stem cell-mediated immunosuppression occurs via concerted action of chemokines and nitric oxide. Cell Stem Cell, 2, 141-150.

10. Tipnis, S., Viswanathan, C., \& Majumdar, A. S. (2010). Immunosuppressive properties of human umbilical cord-derived mesenchymal stem cells: role of B7-H1 and IDO. Immunology and Cell Biology, 88, 795-806.

11. Davies, L. C., Heldring, N., Kadri, N., \& Le Blanc, K. (2017). Mesenchymal stromal cell secretion of programmed death-1 ligands regulates $\mathrm{T}$ cell mediated immunosuppression. Stem Cells, 35, 766-776.

12. Le Blanc, K., Frassoni, F., Ball, L., et al. (2008). Mesenchymal stem cells for treatment of steroid-resistant, severe, acute graftversus-host disease: a phase II study. Lancet., 371, 1579-1586.

13. Ringden, O., Uzunel, M., Rasmusson, I., et al. (2006). Mesenchymal stem cells for treatment of therapy-resistant graftversus-host disease. Transplantation., 81, 1390-1397.

14. Rizk, M., Monaghan, M., Shorr, R., Kekre, N., Bredeson, C. N., \& Allan, D. S. (2016). Heterogeneity in studies of mesenchymal stromal cells to treat or prevent graft-versus-host disease: a scoping review of the evidence. Biology of Blood and Marrow Transplantation, 22, 1416-1423.

15. Galipeau, J., \& Sensébé, L. (2018). Mesenchymal stromal cells: clinical challenges and therapeutic opportunities. Cell Stem Cell, $22,824-833$.

16. Théry, C., Witwer, K. W., Aikawa, E., et al. (2018). Minimal information for studies of extracellular vesicles 2018 (MISEV2018): a position statement of the International Society for Extracellular Vesicles and update of the MISEV2014 guidelines. The Journal of Extracellular Vesicles, 7, 1535750.

17. Van Niel, G., D'Angelo, G., \& Raposo, G. (2018). Shedding light on the cell biology of extracellular vesicles. Nature Reviews. Molecular Cell Biology, 19, 213-228.

18. Kordelas, L., Rebmann, V., Ludwig, A.-K., Radtke, S., Ruesing, J., Doeppner, T. R., Epple, M., Horn, P. A., Beelen, D. W., \& Giebel, B. (2014). MSC-derived Exosomes: a novel tool to treat therapyrefractory graft-versus-host disease. Leukemia., 28, 970-973.

19. Allan, D.S., Tieu, A., Lalu, M., \& Burger, D. (2019). Mesenchymal stromal cell-derived extracellular vesicles for regenerative therapy and immune modulation: Progress and challenges. Stem Cells Translational Medicine. (epub ahead of print Aug 14, 2019).

20. Tieu, A., Slobodian, M., Fergusson, D. A., Montroy, J., Burger, D., Stewart, D. J., Shorr, R., Allan, D. S., \& Lalu, M. M. (2019). Methods and efficacy of extracellular vesicles derived from mesenchymal stromal cells in animal models of disease: a preclinical systematic review protocol. Systematic Reviews., 8, 322.

21. Hooijmans, C. R., Rovers, M. M., de Vries, R. B. M., Leenaars, M., Ritskes-Hoitinga, M., \& Langendam, M. W. (2014). SYRCLE's risk of bias tool for animal studies. BMC Medical Research Methodology, 14, 43.

22. Dominici, M., Le Blanc, K., Mueller, I., et al. (2006). Minimal criteria for defining multipotent mesenchymal stromal cells. The International Society for Cellular Therapy Position Statement. Cytotherapy., 8, 315-317.

23. Fujii, S., Miura, Y., Fujishiro, A., Shindo, T., Shimazu, Y., Hirai, H., Tahara, H., Takaori-Kondo, A., Ichinohe, T., \& Maekawa, T. (2018). Graft-versus-host disease amelioration by human bone marrow mesenchymal stromal/stem cell-derived extracellular vesicles is associated with peripheral preservation of naive T cell populations. Stem Cells, 36, 434-445.

24. Lai, P., Chen, X., Guo, L., et al. (2018). A potent immunomodulatory role of exosomes derived from mesenchymal stromal cells in preventing CGVHD. The Journal of Hematology and Oncology, 1, 135.

25. Zhang, B., Yeo, R. W. Y., Lai, R. C., Sim, E. W. K., Chin, K. C., \& Lim, S. K. (2018). Mesenchymal stromal cell exosome-enhanced regulatory T-cell production through an antigen-presenting cell-mediated pathway. Cytotherapy., 20, 687-696.

26. Wang, L., Gu, Z., Zhao, X., Yang, N., Wang, F., Deng, A., Zhao, S., Luo, L., Wei, H., Guan, L., Gao, Z., Li, Y., Wang, L., Liu, D., \& Gao, C. (2016). Extracellular vesicles released from human umbilical cord-derived mesenchymal stromal cells prevent lifethreatening acute graft-versus-host disease in a mouse model of allogeneic hematopoietic stem cell transplantation. Stem Cells and Development, 25, 1874-1883.

27. Van Deun, J., Mestdagh, P., Sormunen, R., et al. (2014). The impact of disparate isolation methods for extracellular vesicles on downstream RNA profiling. The Journal of Extracellular Vesicles, 3. https://doi.org/10.3402/jev.v3.24858.

28. Busatto, S., Vilanilam, G., Ticer, T., Lin, W. L., Dickson, D., Shapiro, S., Bergese, P., \& Wolfram, J. (2018). Tangential flow filtration for highly efficient concentration of extracellular vesicles from large volumes of fluid. Cells., 7, 273.

29. Lia, G., Brunello, L., Bruno, S., Carpanetto, A., Omedè, P., Festuccia, M., Tosti, L., Maffini, E., Giaccone, L., Arpinati, M., Ciccone, G., Boccadoro, M., Evangelista, A., Camussi, G., \& Bruno, B. (2018). Extracellular vesicles as potential biomarkers of acute graft-vs-host disease. Leukemia., 32, 765-773.

30. Lia, G., Di Vito, C., Cerrano, M., et al. (2020). Extracellular vesicles after allogeneic hematopoietic cell transplantation: emerging role in post-transplant complications. Frontiers in Immunology, 11,422 .

31. Lavoie, J. R., \& Rosu-Myles, M. (2013). Uncovering the secretes of mesenchymal stem cells. Biochimie., 95, 2212-2221.

32. Bruno, S., Deregibus, M. C., \& Camussi, G. (2015). The secretome of mesenchymal stromal cells: role of extracellular vesicles in immunomodulation. Immunology Letters, 168, 154-158.

33. Blazquez, R., Sanchez-Margallo, F. M., de la Rosa, O., et al. (2014). Immunomodulatory potential of human adipose mesenchymal stem cells derived exosomes on in vitro stimulated $\mathrm{T}$ cells. Frontiers in Immunology, 5, 556.

34. Park, H., Park, H., Mun, D., Kang, J., Kim, H., Kim, M., Cui, S., Lee, S. H., \& Joung, B. (2018). Extracellular vesicles derived from hypoxic human mesenchymal stem cells attenuate GSK $3 \beta$ expression via MiRNA-26a in an ischemia-reperfusion injury model. Yonsei Medical Journal, 59, 736-745.

35. Zhu, L. P., Tian, T., Wang, J. Y., He, J. N., Chen, T., Pan, M., Xu, L., Zhang, H. X., Qiu, X. T., Li, C. C., Wang, K. K., Shen, H., Zhang, G. G., \& Bai, Y. P. (2018). Hypoxia-elicited mesenchymal stem cell-derived exosomes facilitates cardiac repair through MiR$125 \mathrm{~b}$-mediated prevention of cell death in myocardial infarction. Theranostics., 8, 6163-6177.

36. Kadle, R. L., Abdou, S. A., Villarreal-Ponce, A. P., Soares, M. A., Sultan, D. L., David, J. A., Massie, J., Rifkin, W. J., Rabbani, P., \& Ceradini, D. J. (2018). Microenvironmental cues enhance mesenchymal stem cell-mediated immunomodulation and regulatory Tcell expansion. PLoS One, 13, e0193178-e0193178.

37. Henderson, V. C., Kimmelman, J., Fergusson, D., Grimshaw, J. M., \& Hackam, D. G. (2013). Threats to validity in the design and conduct of preclinical efficacy studies: a systematic review of guidelines for in vivo animal experiments. PLoS Medicine, 10, e1001489.

Publisher's Note Springer Nature remains neutral with regard to jurisdictional claims in published maps and institutional affiliations. 